\title{
An Immunocompetent Patient with Isolated Cryptococcosis of the Nasal Cavity: Surgical Treatment without Systemic Antifungal Agents
}

\author{
${ }^{1}$ Seong K Park, ${ }^{2}$ Do H Kim
}

\begin{abstract}
The mode of cryptococcal transmission to humans is believed to be through inhalation of aerosolized droppings, which cause asymptomatic pulmonary granulomata in immunocompetent patients. Extrapulmonary infections have been associated with various immunocompromised states, and these infections are relatively rare in immunocompetent patients. In general, cryptococcal sinus infections are treated with surgical intervention and administration of systemic antifungal agents. However, there is doubt that systemic antifungal agents are necessary in immunocompetent patients with no complications. We report an otherwise healthy woman with cryptococcosis of the nasal cavity who was treated surgically without systemic antifungal agents.
\end{abstract}

Keywords: Antifungal agent, Cryptococcosis, Nasal cavity.

How to cite this article: Park SK, Kim DH. An Immunocompetent Patient with Isolated Cryptococcosis of the Nasal Cavity: Surgical Treatment without Systemic Antifungal Agents. Clin Rhinol An Int J 2017;10(1):36-38.

\section{Source of support: Nil}

Conflict of interest: None

\section{INTRODUCTION}

Cryptococcus neoformans is a yeast with a global distribution. It thrives in soil and is abundant in pigeon excreta. Pigeons, however, are not infected by the organisms and do not serve as carriers. There is no direct pigeonto-human transmission of infection. ${ }^{1}$ The most common site of cryptococcal infection is pulmonary because the yeast exists as a 2 to $7 \mu \mathrm{m}$, capsular, airborne form that is easily inhaled.

Extrapulmonary infection has been associated with various immunocompromised states, including sarcoidosis, Hodgkin's disease, solid tumors, acquired

\footnotetext{
${ }^{1}$ Professor, ${ }^{2}$ Assistant Professor

1,2Department of Otorhinolaryngology - Head and Neck Surgery Inje University College of Medicine, Busan Paik Hospital, Busan Republic of Korea

Corresponding Author: Seong K Park, Professor, Department of Otorhinolaryngology - Head and Neck Surgery, Inje University College of Medicine, Busan Paik Hospital, Busan, Republic of Korea, e-mail: Sinus4@paik.ac.kr
}

immunodeficiency syndrome, and systemic steroid use. ${ }^{1}$ These infections are relatively rare in immunocompetent patients. $^{2}$

Most patients needed to receive systemic antifungal agents for long periods. Indeed, there is no report of cryptococcal sinus infection treated surgically without the use of systemic antifungal agents. ${ }^{3}$ Here, we present the case of an otherwise healthy woman who had no history of pulmonary infection or meningitis with isolated cryptococcosis of the nasal cavity who underwent surgery without systemic antifungal agents.

\section{CASE REPORT}

A 53-year-old woman was referred to our Otolaryngology Department for evaluation of foul-smelling rhinorrhea in the left nasal cavity, which became evident 2 weeks earlier.

Endoscopic examination revealed yellow-black fungal ball-like material and granulation tissue on the left middle meatus (Fig. 1A). The remainder of the head and neck was normal. Laboratory test results were within normal limits. The patient had no history of systemic disease or specific medication. She had no pets, no exposure to animals, and no experience of recent foreign travel.

Paranasal sinus computed tomography (CT) with contrast showed a nonenhancing mass within the left middle meatus and maxillary sinusitis (Fig. 1B).

The mass was resected endoscopically with the patient under general anesthesia. A left uncinectomy was performed to obtain good exposure. The fungus balllike material and granulation tissue on the left middle meatus were removed completely. After the removal of the bulla ethmoidalis and widening of the antrostomy endoscopically, the maxillary sinus was explored with a $30^{\circ}$ endoscope. Some pus was found in the maxillary sinus, but there was no fungal material. There was no significant intraoperative bleeding and the postoperative course was uneventful.

Hematoxylin-eosin staining showed numerous Cryptococcus yeasts in tissue obtained from the nasal cavity (Fig. 2A). We also recognized yeast capsules with mucicarmine staining (Fig. 2B). These findings are compatible with the presence of Cryptococcus sp. 

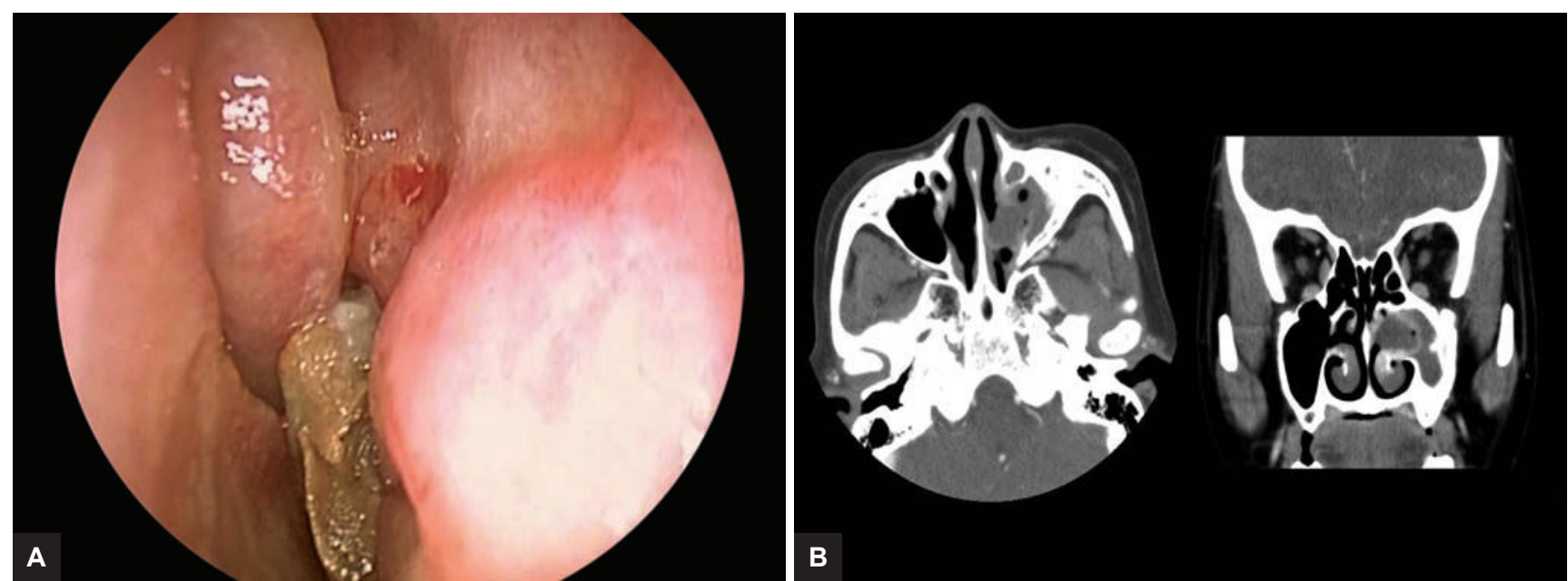

Figs 1A and B: (A) The endoscopic examination showed yellow-black fungal material in the left middle meatus; and (B) axial and coronal paranasal CT with contrast showed a nonenhancing mass in the left middle meatus and maxillary sinusitis
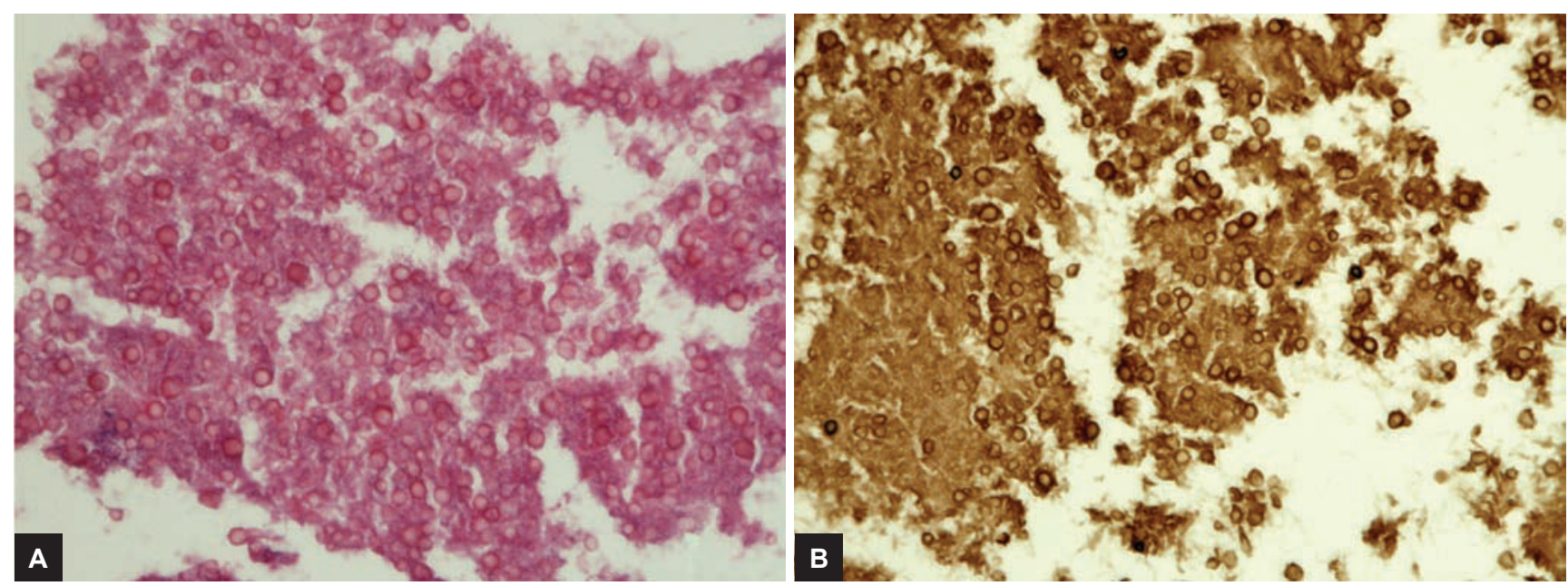

Figs 2A and B: (A) Numerous Cryptococcus yeasts are noted in tissue obtained from the nasal cavity (hematoxylin and eosin $400 \times)$; and (B) typical yeast capsules are seen with mucicarmine staining (400×)

Postoperatively, no antifungal agents, such as fluconazole were administered to this patient. For 2 weeks, cephalosporin antibiotics were administered to prevent secondary bacterial infection, and then low-dose roxithromycin (150 mg/day) was administered for the next 2 months to treat any biofilm. Regular follow-up was performed with angled endoscopy. The postoperative course has been uneventful in 60 months of follow-up. Institutional Review Board approval to review patient data was obtained.

\section{DISCUSSION}

Cryptococcus neoformans is a budding yeast found worldwide in soil, milk, wood, and avian feces. The mode of cryptococcal transmission to humans is believed to be through inhalation of aerosolized droppings, which cause asymptomatic pulmonary granulomata in immunocompetent patients. ${ }^{4}$ Diagnosis is by culture or biopsy.
The hallmark of Cryptococcus is its polysaccharide capsule, which can be up to three times the width of the cell. Specialized diagnostic stains are recommended for cryptococcal infection, such as methenamine silver, periodic acid-Schiff, mucicarmine, and Alcian blue. ${ }^{5}$

Recommended therapy for systemic or extrapulmonary cryptococcal infections has been combination therapy with intravenous amphotericin B or fluconazole, followed by oral fluconazole or 5 -flucytosine. ${ }^{6}$ If central nervous system disease is ruled out, fungemia is not present, infection occurs at a single site, and there are no immunosuppressive risk factors, consider fluconazole [ $400 \mathrm{mg}(6 \mathrm{mg} / \mathrm{kg})$ per day orally] for 6 to 12 months. ${ }^{3}$

There are reports of cryptococcal sinusitis in immunocompromised patients or complications of cryptococcal sinusitis in immunocompetent patients. ${ }^{4,7}$ All of these cases of cryptococcal sinusitis have been treated with antifungal drugs. There are no evidence-based guidelines for the management of cryptococcosis of the nasal cavity 
in immunocompetent patients without complications. However, patients in whom isolated pulmonary cryptococcosis has been excised surgically, who are asymptomatic, and who have no serum cryptococcal antigen titers may or may not require antifungal therapy. ${ }^{3}$

In our case, the antifungal agents were not used because the patient did not have an immunological problem or complications of sinusitis. In noninvasive fungal sinus diseases, surgical treatment, usually via an endonasal endoscopic approach, is curative. ${ }^{9}$ As we know, this is the first successful case who was treated surgically with no administration of antifungal agents in Asia. However, antifungal therapy should be considered in immunocompromised patients or those with complications of cryptococcal sinusitis.

We suggest that antifungal agents are not recommended in isolated cryptococcosis of the nasal cavity in immunocompetent patients with no complications of cryptococcal sinusitis.

\section{REFERENCES}

1. Isaacson JE, Frable MA. Cryptococcosis of the larynx. Otolaryngol Head Neck Surg 1996 Jan;114(1):106-109.
2. Woodring JH, Ciporkin G, Lee C, Worm B, Woolley S. Pulmonary cryptococcosis. Semin Roentgenol 1996 Jan;31(1): 67-75.

3. Prendiville S, Bielamowicz SA, Hawrych A, Deeb ZE. Isolated cryptococcal sphenoid sinusitis with septicemia, meningitis, and subsequent skull base osteomyelitis in an immunocompetent patient. Otolaryngol Head Neck Surg 2000 Sep;123(3):277-279.

4. Choo MJ, Yang SK, Jin HR, Lee OJ. Localized cryptococcal infection combined with cholesteatoma of the ear. Otolaryngol Head Neck Surg 2002 Apr;126(4):453-454.

5. Drouhet, E.; Dupont, B. Cryptococcosis. In: Jacobs, PH.; Nall, L., editors. Antifungal drug therapy. 1st ed. New York: Marcel Dekker, Inc.; 1990. p. 143-160.

6. Gordon DH, Stow NW, Yapa HM, Bova R, Marriott D. Laryngeal cryptococcosis: clinical presentation and treatment of a rare cause of hoarseness. Otolaryngol Head Neck Surg 2010 Mar;142(3 Suppl 1):S7-S9.

7. Choi SS, Lawson W, Bottone EJ, Biller HF. Cryptococcal sinusitis: a case report and review of literature. Otolaryngol Head Neck Surg 1988 Oct;99(4):414-418.

8. Younis RT, Anand VK, Childress C. Sinusitis complicated by meningitis: current management. Laryngoscope 2001 Aug;111(8):1338-1342.

9. Grosjean P, Weber R. Fungus balls of the paranasal sinuses: a review. Eur Arch Otorhinolaryngol 2007 May;264(5): 461-470. 